\title{
Obtenção e caracterização de farinhas de caroço de açaí (Euterper Oleracea) e de casca de bacaba (Oenocarpus Bacaba)
}

Obtaining and characterizing açaí seed (Euterper Oleracea) and bacaba peel (Oenocarpus Bacaba)

flors

Obtención y caracterización de harinas de semilla de açaí (Euterper Oleracea) y piel de bacaba (Oenocarpus bacaba)

Denise Gomes Alves

ORCID: https://orcid.org/0000-0002-5105-3618 Universidade Federal de Alfenas, Brasil E-mail: denise@mail.uft.edu.br

Rodolfo Castilho Clemente

ORCID: https://orcid.org/0000-0002-0766-9968 Universidade Federal do Tocantins, Brasil

E-mail: castilho@mail.uft.edu.br

Douglas Martins da Costa

ORCID: https://orcid.org/0000-0002-8798-6444 Universidade Federal do Tocantins, Brasil

E-mail: douglasbio904@gmail.com

\section{Resumo}

As frutas oriundas dos biomas Amazônia e Cerrado, como açaí (Euterper oleracea) e a bacaba (Oenocarpus bacaba), são apreciadas por seus sabores exóticos, potencial nutricional e funcional. À busca por alimentos saudáveis e a descoberta de nutrientes de cunho funcionais existentes nos resíduos frutícolas, fez com que aumentasse a relevância por subprodutos derivados das cascas e caroços ou sementes, levando para o crescimento da indústria alimentícia. Logo, o objetivo do estudo foi produzir farinha a partir do caroço de açaí e da casca da bacaba, bem como sua caracterização física, química e tecnológica. O caroço de açaí foi adquirido na cidade de Imperatriz (MA) e a bacaba foi obtida na Aldeia Pedra Branca Terra Indígena Krahô no estado do Tocantins. Os resíduos foram secos em estufa com circulação de ar, na temperatura de $50^{\circ} \mathrm{C}$. As farinhas oriundas do caroço de açaí e da casca da bacaba apresentaram valores consideráveis de nutrientes, sendo uma boa fonte de proteínas, lipídios e carboidratos, além de apresentar baixa umidade e $\mathrm{pH}$ ácido contribuindo como barreira contra o crescimento de microrganismos. Os valores de cinza obtidos em ambas as farinhas estão de acordo com a legislação brasileira. A farinha do caroço de açaí e da casca da bacaba apresentaram baixa luminosidade e os valores de chroma $\left(\mathrm{C}^{*}\right)$ e ângulo de hue (h*) indicaram uma coloração avermelhada para todas as farinhas. Os resultados das análises tecnológicas, como índice de absorção e de solubilidade, mostram potencial para elaboração de novos alimentos.

Palavras-chave: Amazônia; Cerrado; Resíduos agroindustriais; Tecnologia de alimentos.

\footnotetext{
Abstract

Those from the Amazon and Cerrado biomes, such açaí (Euterper fruit oleracea) and bacaba (Oenocarpus bacaba), are appreciated for their exotic flavors, nutritional and functional potential. The search for healthy foods and the discovery of functional nutrients existing in fruit residues, has increased the relevance of by-products derived from
} 
peels and seeds or seeds, leading to the growth of the food industry. Therefore, the objective of the study was to produce flour from the açaí core and the peel bacaba, as well its physical, chemical and technological characterization. The açaí seed was purchased in the city of Imperatriz (MA) and the bacaba was obtained in the village of Pedra Branca Terra Indígena Krahô in the state of Tocantins. The residues were dried in an oven with air circulation, at a temperature of $50^{\circ} \mathrm{C}$. Flours from açaí stone and bacaba peel presented considerable values of nutrients, being a good source of proteins, lipids and carbohydrates, besides presenting low humidity and acidic $\mathrm{pH}$ contributing as a barrier against the growth of microorganisms. The gray values obtained in both flours are in accordance with Brazilian legislation. The flour from the açaí core and the bacaba peel showed low luminosity and the chroma $(\mathrm{C} *)$ and hue angle $(\mathrm{h} *)$ values indicated a reddish color for all flours. The results of technological analysis, such as absorption and solubility index, show potential for the elaboration of new foods.

Keywords: Amazon; Cerrado; Agro-industrial waste; Food technology.

\section{Resumen}

Las frutas de los biomas del Amazonas y Cerrado, como el açaí (Euterper oleracea) y la bacaba (Oenocarpus bacaba), son apreciadas por sus sabores exóticos, su potencial nutricional y funcional. La búsqueda de alimentos saludables y el descubrimiento de nutrientes funcionales existentes en los residuos de frutas, ha incrementado la relevancia de los subproductos derivados de las cáscaras y semillas o semillas, lo que ha llevado al crecimiento de la industria alimentaria. Por tanto, el objetivo del estudio fue producir harina a partir del núcleo de açaí y la cáscara de bacaba, así como su caracterización física, química y tecnológica. La semilla de açaí se compró en la ciudad de Imperatriz (MA) y la bacaba se obtuvo en el pueblo de Pedra Branca Terra Indígena Krahô en el estado de Tocantins. Los residuos se secaron en estufa con circulación de aire, a una temperatura de $50^{\circ} \mathrm{C}$. Los valores de gris obtenidos en ambas harinas están de acuerdo con la legislación brasileña. La harina de la semilla de açaí y la cáscara de bacaba mostraron baja luminosidad y los valores de croma $(\mathrm{C} *)$ y ángulo de matiz $(\mathrm{h} *)$ indicaron un color rojizo para todas las harinas. Los resultados del análisis tecnológico, como el índice de absorción y solubilidad, muestran potencial para la elaboración de nuevos alimentos.

Palabras clave: Amazonia; Cerrado; Residuos agroindustriales; Tecnología alimentaria.

\section{Introdução}

De acordo com a RDC n 263, de 22 de setembro de 2005 (Brasil, 2005a), as farinhas são produtos obtidos de partes comestíveis de espécies de cereais, leguminosas, frutos, sementes, tubérculos e rizomas por moagem e/ou vários outros processos tecnológicos considerados seguros para produção de alimentos.

$\mathrm{O}$ aumento na demanda por ingredientes que desempenham um papel importante no organismo, além de promover a redução de volume de resíduos sólidos, proporcionando seu reaproveitamento, cresce cada vez mais no meio das indústrias alimentícias (Páramo-Calderón et al, 2019 ). Neste sentido, as farinhas vegetais são consideradas um dos veículos mais apropriados no ramo alimentício devido ao seu baixo custo, além de conter múltiplos nutrientes. As mesmas podem ser usadas como alternativas na substituição da farinha de trigo, para compor farinhas mistas na fabricação de produtos de panificação, como biscoitos e pães, e massas alimentícias, além de reduzir os impactos ambientais e proporcionar os benefícios para a saúde humana (Saubade, et al, 2017; Silveira et al, 2016).

O açaí e a bacaba são frutas exóticas encontradas nos biomas Amazônia e Cerrado, importantes para população regional por serem fontes de vitaminas, minerais, fibras e possuírem alta atividade antioxidante. Estes nutrientes estão presentes não apenas nas polpas, mas em outras partes envolvidas do fruto que são frequentemente descartadas (Schiassi et al, 2018; Sampaio et al, 2020).

O caroço do açaí compõe a maior parte do fruto correspondendo a $85 \%$ do peso total, e o mesmo é comumente descartado de forma indevida, porém poderia ser reaproveitado para elaboração de alimentos, pois se mostra uma alternativa barata para a retirada de fibras, proteínas, minerais e polifenóis (Wycoff et al, 2015; Rodriguez et al, 2006). Já a casca da bacaba, além de apresentar substâncias bioativas, alto teor de lipídeos, antioxidante naturais, ainda exibe uma coloração que indica a presença de antocianinas pertencente ao grupo flavonóides (Abadio Finco, et al, 2012). Essas partes residuais orgânicas podem ser reaproveitadas e colaborar na preservação do equilíbrio ambiental. 
À busca por alimentos saudáveis e a descoberta de nutrientes de cunho funcionais existentes nos resíduos frutícolas, fez com que aumentasse a relevância por subprodutos derivados das cascas e caroços ou sementes, levando para o crescimento da indústria alimentícia (Durante et al, 2017; Gerhardt, et al, 2012).

Para aplicação das farinhas de subprodutos, as mesmas precisam ser analisadas quanto o seu desempenho como ingredientes funcionais, comportamento tecnológico em determinados sistemas alimentares durante o processo da elaboração do produto e averiguar sua qualidade final, antes da sua aplicação (Guimarães et al, 2012; Tavares et al, 2012). Diante disso, o objetivo do trabalho foi elaborar farinhas a partir do caroço de açaí e da casca da bacaba e caracterizá-las quanto às análises físico-químicas e tecnológicas.

\section{Metodologia}

\subsection{Tipo de estudo}

Trata-se de uma pesquisa explicativa e experimental, com parte conduzida no espaço campestre onde os frutos foram colhidos e nos laboratórios de tecnologia de alimentos, análises de alimentos (LANA) e de frutas e hortaliças (LAFRUTEC), da universidade federal do Tocantins, campus palmas, sendo de natureza quantitativa de acordo com pereira et al. (2018).

\subsection{Matéria-prima}

Os caroços de açaí foram adquiridos na cidade de Imperatriz (MA) em 16 de agosto de 2019, e em seguida transportados para Laboratório de Tecnologia de Alimentos da Universidade Federal do Tocantins, campus Palmas (TO). Já os frutos da bacaba foram adquiridos na Aldeia Pedra Branca Terra Indígena Krahô Itacajá - TO. No momento da colheita, as bacabas foram retiradas por cacho, e em seguida, armazenadas em saco plástico. A preparação do experimento ocorreu dentro de um período de 24 horas após a retirada dos frutos.

Os caroços de açaí e as bacabas passaram por um processo de seleção inicial, após a seleção os mesmos foram colocados numa solução de hipoclorito de sódio a 200 por 15 minutos e, em seguida foram lavados em água corrente para retirar o excesso de cloro, de acordo com a Resolução da Agência de Vigilância Sanitária - RDC nº 216/2004 (Brasil, 2004). Os caroços foram embalados em sacos de polipropileno e congelados a $-18^{\circ} \mathrm{C}$ até o uso. Para obtenção das cascas do fruto da bacaba o despolpamento foi feito manualmente. As frutas foram submersas em água ultra-pura a $80^{\circ} \mathrm{C}$ por 30 minutos para remoção manual das cascas, o material removido foi levado imediatamente para estufa.

\subsection{Obtenção e preparo das farinhas de caroço de açaí e da casca da bacaba}

Os caroços do açaí e as cascas das bacabas foram acomodados em bandejas e levados para estufa de circulação forçada de ar do modelo (SL -102) marca Ethik e secas a $50^{\circ} \mathrm{C}$, até $15 \%$ de umidade, conforme a RDC 263/2005 (Brasil, 2005). Os caroços foram secos no período de 28 horas e as cascas das bacabas por 45 horas.

Os caroços secos foram triturados, em porç̃̃es de 50g, utilizando um moinho de facas, tipo Willye (modelo STAR FT- 50) por 45s, após serem triturados foram passados em moinho de martelo (Vieira MCS 280 (05 cv)) nas peneiras de furo de $0,7 \mathrm{~mm}$ e de $0,3 \mathrm{~mm}$. As cascas, após a secagem, foram trituradas usando um liquidificador (Mondial Turbo, L$1200 \mathrm{BI}$ ) por cerca de 30s. Posteriormente, as cascas trituradas foram peneiradas e, em seguida foram submetidas à moagem em moinho de martelo (Vieira MCS $280(05 \mathrm{cv})$ ) com a peneira do furo $0.3 \mathrm{~mm}$. Os produtos do resíduo retirado das peneiras foram classificados como farinhas (Figura 1). 
Figura 1: Farinha (1) do caroço de açaí e Farinha (2) da casca da bacaba.

1)

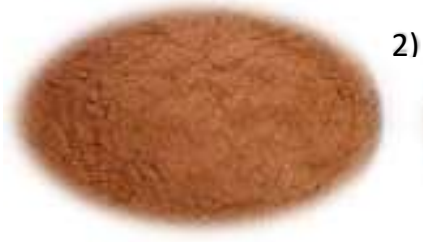

2)

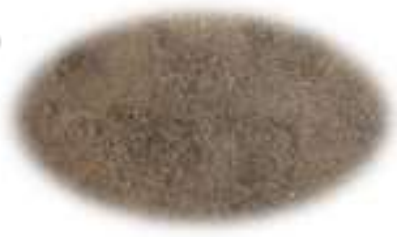

Fonte: Autores.

A farinha do caroço de açaí (FCA) foi acondicionada em sacos plásticos de média densidade (PEMD), de 1 kg, seladas a quente em seladora comercial e armazenada, respectivamente, à temperatura ambiente. Para a farinha do resíduo da bacaba (FCB) foi fracionada em porções de 100g e acondicionada em saco Stand Up Pouch Metalizado, selado a quente em seladora comercial e armazenado dentro do dessecador. Em razão de sua alta quantidade de lipídeos, a farinha da casca de bacaba foi armazenada de forma a impossibilitar a rancidez oxidativa.

\subsection{Caracterização físico-químicas, composição proximal e cor das farinhas FCA e FCB}

As análises físico-químicas, composição proximal e os parâmetros colorimétricos foram realizados em triplicatas com três repetições.

\subsection{1 pH, acidez total titulável e sólidos solúveis ( $\left.{ }^{\circ} \mathrm{Brix}\right)$}

Para a determinação do $\mathrm{pH}$ as farinhas FCA e FCB foram dispersas em água destilada, com auxílio de um potenciômetro digital da marca DigimedR modelo DM-22. A acidez titulável (TA, g ácido cítrico 100 g-1) foi determinada por titulação, utilizando $0,1 \mathrm{~N}$, ambos parâmetros foram recomendados de acordo com (AOAC, 2012). Já para determinação do ${ }^{\circ}$ Brix as amostras foram diluídas com água destilada e filtradacom auxílio de papel de filtro qualitativo em erlenmeyers de $125 \mathrm{~mL}$ a leitura do ${ }^{\circ}$ Brix foi executada no medidor de concentração - DMA 35 modelos A- 8054 Graz em base úmida de acordo com (AOAC, 2012).

\subsubsection{Composição proximal}

As FCA e FCB foram caracterizadas quanto os teores de umidade utilizando o método gravimétrico, através da perda de peso da amostra submetida a aquecimento em estufa a $50^{\circ} \mathrm{C}$, até a obtenção de peso constante do produto dessecado de acordo Association of Official Analytical Chemists International (AOAC, 2005). As demais análises de proteína, cinzas e lipídios também foram determinadas de acordo com a AOAC (2012). A quantificação da fibra bruta foi realizada pelo método gravimétrico com digestão ácida da amostra com ácido sulfúrico $\left(\mathrm{H}_{2} \mathrm{SO}_{4}\right)$, de acordo com a AOAC (2000). Os valores de fração disponíveis foram quantificados por diferenças. O valor energético total (VET) foi estimado em kcal/100g com os fatores de conversão Atwater e Woods (1906), 4kcal/g para carboidratos, 4 kcal/g para proteínas e 9 $\mathrm{kcal} / \mathrm{g}$ para lipídios segundo Brasil (2003).

\subsubsection{Análise de cor}

A cor da farinha FCA e FCB foi determinada em leitura direta usando colorímetro (Konica Minolta Sensing, modelo CR400). A região analisada foi a parte superior. Com determinação no modo CIEL* a* e b*. Foram também analisados em leitura direta croma $C^{*}$, refere-se saturação ou intensidade da cor e ângulo Hue ( $\left.\mathrm{H}^{*}\right)$, que define a tonalidade cromatica. (Araujo et al, 2015). 


\subsection{Propriedades tecnológicas das farinhas}

\subsection{1 Índice de absorção de água e óleo e solubilidade em água}

O índice de absorção em água (IAA), óleo (IAO) e a solubilidade em água (ISA), das farinhas do caroço de açaí e da casca da bacaba foram determinadas segundo Okezie \& Bello (1988) com adaptações. Uma suspensão com 12,5 mL de água e $0,25 \mathrm{~g}$ de farinhas foram preparadas em tubos de centrifugas com tampa, e submetidas a agitação por 1 minuto. Em seguida as amostras foram centrifugadas a 2650rpm por 10 minutos em centrifuga microprocessada da marca QUIMIS.

Para determinação do ISA, verteu-se cuidadosamente o líquido sobrenadante em placa de alumínio previamente tarada e, em seguida, foi levada para estufa com circulação de ar MD SL-02, a $105^{\circ} \mathrm{C}$, durante 6 horas, até peso constante. Após o período, a amostra foi resfriada em dessecador e pesado em balança analítica digital. O ISA foi calculado pela relação entre a massa evaporada (resíduo seco do sobrenadante) e o peso da amostra.

Para a determinação do índice de absorção de óleo (IAO) a água foi substituída pelo óleo de soja. Após a centrifugação, o líquido sobrenadante de cada amostra foi descartado, deixando-se os tubos ligeiramente invertidos durante 1 min. O IAO foi obtido pela relação entre a massa do resíduo centrifugado pela amostra da farinha. Os resultados são expressos em $g$ de gel (g de matéria seca) ${ }^{-1}$ e porcentagem, respectivamente.

\subsection{Análise estatística}

Os dados coletados foram tabulados em média e desvio-padrão em planilhas desenvolvidas com o programa Microsoft Excel $^{\circledR}$, versão 365, sendo em seguida, as médias comparadas por Análise de Variância (ANOVA) seguida da de teste HolmSidak, sendo aplicado um nível de significância de p < 0,05, com o auxílio do programa GRAPHPAD PRISM 7,0, de 2016 XLStat (Addinsoft, Paris, França).

\section{Resultados e Discussão}

\subsection{Determinação da composição físico-químicas e proximal}

Os resultados descritos na Tabela 1, indicam as análises físico-químicas e proximal realizadas nas farinhas do caroço de açaí (FCA) e da casca da bacaba (FCB).

Pode-se observar que os valores obtidos de $\mathrm{pH}$ nas duas farinhas, FCA e FCB, não apresentaram diferença significativa. Esses produtos são classificados como produtos ácidos (Baruffaldi \& Oliveira, 1998) e segundo Selani et al. (2014), baixos valores de pH diminuem o crescimento de microrganismos, o risco de reações enzimáticas e não enzimáticas, podendo favorecer conservação das farinhas.

O teor de sólidos solúveis totais para farinha do caroço de açaí foi de 1,4 e para a farinha da casca da bacaba de 1,2, não apresentando diferença estatística. Observa-se que são valores baixos, indicando de baixos teores de açúcar. Araújo et a, (2017) relatou que a qualidade de um produto está relacionada este parâmetro, visto que produtos com uma alta concentração provoca a menor adição de açúcar para obtenção do produto final. Segundo Chaves et al, (2004) o ${ }^{\circ}$ Brix é usado na agroindústria para assegurar o controle da qualidade do produto final. 
Tabela 1. Composição físico-química e proximal da farinha do caroço de açaí (FCA) e da farinha de casca da bacaba (FCB).

\begin{tabular}{lcc}
\hline \multicolumn{1}{c}{ Parâmetros } & FCA & FCB \\
\hline & & \\
& & \\
pH & $5,27^{\mathrm{a}} \pm 0,02$ & $4,53^{\mathrm{a}} \pm 0,13$ \\
Sólidos Solúveis $\left(\mathrm{Brix}^{\circ}\right)$ & $1,40^{\mathrm{a}} \pm 0,00$ & $1,20^{\mathrm{a}} \pm 0,00$ \\
Acidez titulável total $(\%)$ & $0,43^{\mathrm{a}} \pm 0,01$ & $1,19^{\mathrm{a}} \pm 0,02$ \\
Umidade $(\%)$ & $8,91^{\mathrm{a}} \pm 0,20$ & $5,21^{\mathrm{b}} \pm 0,26$ \\
Lipídios $\left(g 100 g^{-1}\right)$ & $1,57^{\mathrm{b}} \pm 0,18$ & $22,19^{\mathrm{a}} \pm 0,79$ \\
Proteínas $\left(g 100 g^{-1}\right)$ & $6,65^{\mathrm{b}} \pm 0,06$ & $10,13^{\mathrm{a}} \pm 0,12$ \\
Cinzas $\left(g 100 g^{-1}\right)$ & $1,31^{\mathrm{a}} \pm 0,01$ & $1,47^{\mathrm{a}} \pm 0,00$ \\
Fração Glicídica $\left(g 100 g^{-1}\right)$ & $34,55^{\mathrm{b}} \pm 0,43$ & $48,98^{\mathrm{a}} \pm 0,54$ \\
Valor energético total $(\mathrm{kcal})$ & $178,95^{\mathrm{b}} \pm 0,15$ & $432,72^{\mathrm{a}} \pm 0,78$ \\
& &
\end{tabular}

\footnotetext{
* Médias com letras minúsculas diferentes entre as colunas diferem estatisticamente, pelo teste Holm-Sidak de $(\mathrm{p}<0,05)$. Fonte: Autores.
}

Quanto maior a acidez, menor será a qualidade da farinha. De acordo com os resultados encontrados, as farinhas do caroço de açaí e da casca da bacaba não apresentaram diferença estatística. Ambas as farinhas estão dentro do padrão estabelecido pela ANVISA (1996) que apresenta valores máximo 2\% para acidez.

Para determinação de umidade os resultados encontrados neste trabalho foram de 8,91\% para FCA e 5,21\% para FCB, indicando diferença significativa. No entanto, ambas farinhas estão dentro dos padrões estabelecidos pela RDC $\mathrm{n}^{\circ} 263$ de 22 de setembro de 2005 (Brasil, 2005) que preconiza no máximo 15\% de teor de água para as farinhas obtidas de frutos e sementes. Ainda que a $a_{w}$ da farinha assegure sua estabilidade microbiológica $\left(a_{w}<0,60\right)$ é necessário que o produto esteja armazenado em condições que desfavoreçam o ganho de umidade, em consequência o aumento da $\mathrm{a}_{\mathrm{w}}$ (Pires et al, 2017).

O teor de lipídio para a farinha do caroço de açaí apresentou um valor muito baixo $\left(1,57 \mathrm{~g} 100 \mathrm{~g}^{-1}\right)$ comparado ao valor da farinha da casca da bacaba $\left(22,19 \mathrm{~g}\left(00 \mathrm{~g}^{-1}\right)\right.$, diferindo estatisticamente entre si $(\mathrm{p}<0,05)$. A farinha da casca apresentou um elevado teor de lipídio resultado similar ao encontrado por Seixas et al. (2016) de 21,02 g/100g indicando ser um alimento rico em lipídios. Corrêa et al. (2019) avaliou a casca da bacaba e encontrou um valor de 29,13g/100g um valor superior ao encontrando, o que pode ser justificado pelo local de produção, do solo e do estado de maturação do fruto. Vale ressaltar que a quantidade de lipídios encontrada na farinha da casca da bacaba é preocupante, pois altos valores podem oxidar o alimento com facilidade e rapidez. Em contrapartida, a determinação de lipídios em farinhas obtidas de resíduos utilizados como ingrediente em formulações alimentícias torna-se interessante, visto que contribui na qualidade do alimento, mediante aos atributos textura, sabor e valor calórico. Neste sentindo, o farináceo obtido do caroço de açaí possui um baixo teor de lipídeos, o que dificultar as reações de rancidez (Fennema et al, 2010).

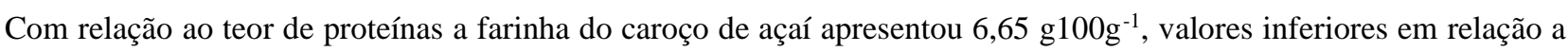

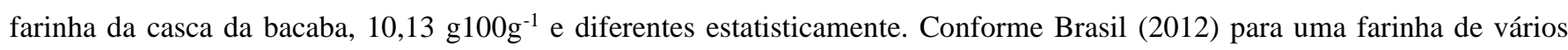
tipos ser considerada rica em proteína precisa ter no mínimo 6g/100g. As farinhas desenvolvidas neste trabalho podem ser consideradas fontes proteicas e de suma importância ao consumo para manutenção fisiológica do nosso organismo (Cozzolino \& Cominetti, 2013).

Para os teores de cinzas, ambas as farinhas, FCA e FCB, não diferem estatisticamente entre si. Os valores encontrados estão de acordo com a legislação (Brasil, 2005), que determina um limite máximo de $2 \%$ de base seca para farinhas. A presença destes minerais nas farinhas indica potencial nutricional, contribuindo para produção de produtos de panificação (Silva et al, 2015). 


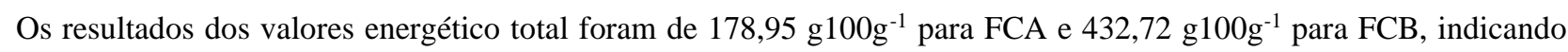
diferença estatística significativa. De acordo com a Anvisa (1998), para que um produto alimentício seja considerado como fonte de energia, ele deve conter $40 \mathrm{kcal}$ por $100 \mathrm{~g}$ sólido, quando os valores encontrados são inferiores a $20 \mathrm{kcal}$ por 100 gramas o alimento é visto como valor baixo calórico. As farinhas estudadas no presente trabalho foram classificadas como um produto de alto valor calórico.

\subsection{Análise de cor das farinhas}

A coloração das farinhas do caroço do açaí e da casca da bacaba e foi avaliada com base nas variáveis luminosidade $\left(L^{*}\right)$, coordenadas $a^{*}$ e $b^{*}$, cromaticidade (chroma) e ângulo Hue $\left(h^{*}\right)$, e os resultados das análises de colorimetria estão expressos na Tabela 2.

Tabela 2. Análise de colorimetria da farinha do caroço do açaí (FCA) e farinha da casca da bacaba (FCB).

\begin{tabular}{|c|c|c|c|c|c|}
\hline \multirow[t]{2}{*}{ Amostras } & \multicolumn{5}{|c|}{$\begin{array}{l}\text { Análise colorimetria } \\
\text { ("Média } \pm \text { DP) }\end{array}$} \\
\hline & $\mathbf{L}^{*}$ & $a^{*}$ & $\mathbf{b}^{*}$ & $\mathbf{H}^{*}$ & $\mathrm{C}^{*}$ \\
\hline FCA & $36,92^{\mathrm{a}} \pm 0,69$ & $2,85^{\mathrm{b}} \pm 0,10$ & $12,67^{\mathrm{a}} \pm 0,18$ & $65,70^{\mathrm{a}} \pm 0,38$ & $22,28^{\mathrm{a}} \pm 0,60$ \\
\hline FCB & $19,65^{\mathrm{b}} \pm 0,48$ & $5,08^{\mathrm{a}} \pm 0,12$ & $7,87^{b} \pm 0,01$ & $58,86^{\mathrm{b}} \pm 0,57$ & $12,77^{\mathrm{b}} \pm 0,38$ \\
\hline
\end{tabular}

* Médias com letras minúsculas diferentes entre as colunas diferem estatisticamente, pelo teste Holm-Sidak de $(\mathrm{p}<0,05)$. Fonte: Autores.

O resultado encontrado para a luminosidade indicou que a FCB se mostrou mais escura do que a FCA. Para a casca da bacaba estudada por Corrêa et al. (2019) no valor $L^{*}$ foi de 19,03 sendo similar com os dados obtidos do presente estudo, afirmando ser uma cor escura. A coordenada $\mathrm{a}^{*}$ para as farinhas apresentaram diferença estatística, enquanto que o parâmetro b* apresentou maiores valores para a FCA.

Na cromaticidade foi encontrado para FCA e FCB os respectivos valores, 22,28 e 12,77, e de acordo com Lawless \& Heymann (1998) quanto mais altos os valores de ${ }^{*} \mathrm{C}$, mais viva é a cor observada, o que indica no presente estudo que FCA possui uma cor mais viva quando comparada a FCB. Os valores de $h^{*}$ indicaram que a FCA é mais avermelhada que a FCB, que se mostrou mais acinzentado.

\subsection{Propriedades tecnológicas das farinhas}

As propriedades tecnológicas das farinhas do caroço de açaí e da casca da bacaba foi realizada pelo Índice de Absorção de Água (IAA), Solubilidade em água (ISA) e Absorção de Óleo (IAO). O conhecimento dessas características torna-se essencial pois permite a inserção destas farinhas na indústria de alimentos e, por decorrência, na dieta humana. Os resultados das propriedades tecnológicas das farinhas avaliadas estão apresentados na Tabela 3. 
Tabela 3. Propriedades tecnológicas das farinhas do caroço de açaí e da casca da bacaba.

\begin{tabular}{cccc}
\hline \multirow{2}{*}{ Amostras } & \multicolumn{3}{c}{$\begin{array}{c}\text { Parâmetro Avaliado } \\
(* \text { Média } \pm \text { DP })\end{array}$} \\
\cline { 2 - 4 } & IAA $(\mathbf{g} / \mathbf{1 0 0 g})$ & ISA(\%) & IAO(g/100g) \\
\hline FCA & $6,53^{\mathrm{a}} \pm 0,63$ & $2,10^{\mathrm{a}} \pm 0,09$ & $12,83^{\mathrm{a}} \pm 0,17$ \\
FCB & $6,52^{\mathrm{a}} \pm 0,50$ & $3,29^{\mathrm{a}} \pm 0,05$ & $8,48^{\mathrm{b}} \pm 0,31$ \\
\hline
\end{tabular}

* Médias com letras minúsculas diferentes entre as colunas diferem estatisticamente, pelo teste Holm-Sidak de (p<0,05). Fonte: Autores.

Os valores IAA encontrados para as farinhas FCB e FCA foram iguais estatisticamente, sendo respectivamente 6,52g e 6,53g. Esses índices são explicados devido a quantidade de fibras que permite capacidade de absorver água. O IAA é um parâmetro benéfico para os produtos de panificação, pois ajuda na incorporação de água à massa e aumenta o rendimento do produto final (Sogi et. al, 2013). Diante deste contexto os resultados de IAA encontrados são de grande relevância.

Para o ISA as farinhas FCA e FCB não deferindo estatisticamente entre si. De acordo com Ferreira et al. (2015) a solubilidade é uma determinante que retrata a deterioração do grânulo de amido. Sampaio (2016), descreve que a solubilidade serve para aperfeiçoar os efeitos do calor nas proteínas durante o processo e quanto maior for o valor de solubilidade para uma proteína, melhor será a sua aplicação na indústria alimentícia. Os valores de ISA encontrados neste trabalho indicam que as farinhas não devem ser empregadas na elaboração de produtos expandidos.

O índice de absorção de óleo (IAO) das farinhas do caroço de açaí e da casca da bacaba, diferiu entre si. Para Padilha et al. (1996), IAO é dado principalmente à combinação de gorduras que estão vinculadas às proteínas presentes, visto que, é nessa estrutura que o óleo permanece retido. Além do mais, essa propriedade verifica sabor e ajuda a melhorar a eficiência do produto (Rodríguez-Ambriz et al, 2005). Neste contexto, a farinha da casca da bacaba por apresentar um valor de 10,13 g100g${ }^{1}$ de proteína mostrou uma capacidade melhor de absorção em relação à farinha do caroço de açaí.

\section{Conclusão}

As farinhas de caroço de açaí e de casca de bacaba apresentaram valores consideráveis de nutrientes, uma boa coloração e indicaram viabilidade para elaboração de produtos de panificação.

\section{Agradecimentos}

Os autores agradecem à coordenação de Aperfeiçoamento de Pessoal de Nível Superior - CAPES, pelo apoio financeiro e à Universidade Federal do Tocantins - Campus Palmas/TO.

\section{Referências}

AOAC (2012). Association of Official Analytical Chemistry. Official methods of analysis international (19a ed.). International Gaithersburg, p, 3000.

AOAC (2000). Association of Official Analytical Chemistry. Official methods of analysis international. International Gaithersburg, p. 982.

Abadio Finco, F. D. B., Kammerer, D. R., Carle, R., Tseng, W. H., Boser, S., \& Graeve, L. (2012). Antioxidant activity and characterization of phenolic compounds from bacaba (Oenocarpus bacaba Mart.) fruit by HPLC-DAD-MS n. Journal of agricultural and food chemistry, 60(31), 7665-7673. doi:10.1021/jf3007689

Araújo, K. T. A, Da Silva, R. M., Da Silva, R. C., De Figueirêdo, R. M. F., \& De Melo Queiroz, A. J. Caracterização físico-química de farinhas de frutas tropicais. Revista Brasileira de Agrotecnologia, 7(2), 110-115.

Araujo, D. R., De Lucena, E. M. P., Gomes, J. P., De Figueirêdo, R. M. F., \& Silva, E. E. (2015). Características físicas, químicas e físico-químicas dos frutos da murta. Revista Verde de Agroecologia e Desenvolvimento Sustentável, 10(3), 11-17. 10.18378/rvads.v10i3.3115 
Baruffaldi, R., \& Oliveira, M. N. (1998). Fatores que condicionam a estabilidade de alimentos. In: Fundamentos de tecnologia de alimentos. São Paulo: Atheneu, 3, 13-25.

Brasil. (2005). Resolução RDC nº 54, de 12 de novembro de 2012. Dispõe sobre o Regulamento Técnico sobre Informação Nutricional Complementar. Diário Oficial da União, Poder Executivo, DF, Brasília, 2012.

Brasil. (2005). Resolução RDC n 263 de 22 de setembro de 2005. Aprova o Regulamento Técnico para produtos de cereais, amidos, farinhas e farelos, constantes do anexo desta Portaria. Diário Oficial União, Brasília, DF.

Brasil. (2004). Ministério da Saúde. Resolução RDC n 216, de 15 de setembro de 2004. Regulamento Técnico de Boas Práticas para Serviços de Alimentação. Diário Oficial da União, Poder Executivo, Brasília, DF.

Brasil. (2003). Resolução RDC n ${ }^{\circ}$ 360, de 23 de dezembro de 2003. Aprova o Regulamento Técnico sobre rotulagem nutricional de alimentos embalados. Diário Oficial [da] República Federativa do Brasil. Brasília, Seção 1, p.4.

Brasil. (1998). Ministério da Saúde. Agência Nacional de Vigilância Sanitária. Portaria nº 27, de 13 de janeiro de 1998. Aprova o regulamento Técnico referente à Informação Nutricional Complementar (declarações relacionadas ao conteúdo de nutrientes). Diário Oficial [da] República Federativa do Brasil, Brasília, DF.

Brasil. (1996). Institui o Regulamento Técnico sobre a identidade e as características mínimas de qualidade a que deverá obedecer a farinha de trigo (Portaria nº 354, de 18 de julho de 1996.). Diário Oficial da República Federativa do Brasil, Brasília, DF.

Chaves, M. C. V., Gomes, J. P., De Almeida, F. A. C., Araujo, L., Cledimario, J., \& Honorato, F. L. Da S. (2004). Caracterização físico-química do suco de acerola. Revista de Biologia e Ciência da Terra, 4(2), 2317-3114.

Correa, B. M., Baldissera, L., Barbosa, F. R., Ribeiro, E. B., Andrighetti, C. R., Da Silva A. J., \& De Sousa V. D. M. (2019). Centesimal and mineral composition and antioxidant activity of the bacaba fruit peel. Bioscience Journal, 35(2). 10.14393/BJ-v35n2a20198-41868.

Cozzolino, S. M. F., \& Cominetti, C. (2013). Bases bioquímicas e fisiológicas da nutrição nas diferentes fases da vida, na saúde e na doença, Ed. Manole, Barueri, Sp, p. 1288.

Durante, M., Montefusco, A., Marrese, P. P., Soccio, M., Pastore, D., Piro, G., \& Lenucci, M. S. (2017). Seeds of pomegranate, tomato and grapes: An underestimated source of natural bioactive molecules and antioxidants from agri-food by-products. Journal of Food Composition and Analysis, 63, 65-72. 10.1016/j.jfca.2017.07.026.

Fennema O. R., Damoradan, S., \& Parkin, K. L. (2010). Química de alimentos de Fennema (4a ed.), Artmed.

Ferreira, M. S. L., Santos, M. C. P., Moro, T. M. A., Basto, G. J., Andrade, R. M. S., Gonçalves, E. C. B. A. (2015). Formulation and characterization of functional foods based on fruit and vegetable residue flour. J Food Sci Technol, 52(2), 822-830.

Gerhardt, C., Wiest, J. M., Girolometto, G., Silva, M. A. S., \& Weschenfelder, S. (2012). Aproveitamento da casca de citros na perspectiva de alimentos: prospecção da atividade antibacteriana. Brazilian Journal of Food Technology, 15, 11-17.

Guimarães, R. C. A., Favoro, S. P., Viana, A. C. A., Braga Neto, J. A., Neves, A V., \& Honer, M. R. (2012). Study of the proteins in the defatted flour and protein concentrate of baru nuts (Dipteryx alata Vog.). Ciência e Tecnologia de Alimentos, Campinas-SP, 32 (3), 464-470. 10.1590/S010120612012005000065 .

Okezie, B. O., \& Bello, A. B. (1988). Physicochemical and functional properties of Winged bean flour and isolate compared with soy isolate. Journal of Food Science, 53(2), 450-454.

Padilla, F. C., Alvarez, M., \& Alfaro, M. J. (1996). Functional properties of barinas nut flour (Caryodendron orinocense Karst., Euphorbiaceae) compared to those of soybean. Food Chemistry,57(2),191-196.

Páramo-Calderón, D. E., Aparicio-Saguilán, A., Aguirre-Cruz, A., Carrillo-Ahumada, J., Hernández-Uribe, J. P., Acevedo-Tello, S., \& Torruco-Uco, J. G. (2019). Tortilla added with Moringa oleifera flour: Physicochemical, texture properties and antioxidant capacity. LWT, 100, 409-415. doi:10.1016/j.1wt.2018.10.078.

Pathare, P. B., Opara, U. L., \& Al-Said, F. A. J. (2013). Colour measurement and analysis in fresh and processed foods: a review. Food and Bioprocess Technology, 6, 36-60.

Pires, F. C. S., Martins, M. G., Cabral, J. D. F., Moraes, W. R. F. B., \& Ena, R. D. S. (2017). Caracterização e Utilização de Farinha de Banana Nanicão (Musa sp.) Semi-madura na produção de um produto de Base Láctea. Revista Brasileira de Produtos Agroindustriais, Campinas Grande, 19(1), 61-72.

Rodríguez-Ambriz, S. L.; Martínez-Ayala, A. L.; Millán, F.; Davila-Ortiz, G. (2005). Composição e propriedades funcionais de isolados de proteína de Lupinus campestres. Plant Foods for Human Nutrition, 60(3) pp. $99-107$.

Rodrigues, R. B., Lichtenthaler, R., Zimmermann, B. F., Papagiannopoulos, H. F., Marx. F., Maia, J. G. S., \& Almeida, O. (2006). Total oxidant scavenging capacity of Euterpe oleracea Mart. (Açaí) seeds and identification of their polyphenolic compounds. J. Agric. Food Chem. 54, 4162-4167. 10.1021/ jf058169p

Sampaio, P. B. R., Pereira, A. S., Pires, C. R. F., Clemente, R. C., \& do Nascimento, G. N. L. (2020). Otimização do processo de extração de antioxidantes presentes na bacaba (oenocarpus distichus mart) utilizando metodologia de superfície de resposta Optimization of the antioxidants extraction process from the bacaba (oenocarpus distichus mart.) using response surface methodology Optimización del proceso de extracción antioxidante presente en bacaba (oenocarpus). Research, Society and Development, 9(8), e229985427. 
Research, Society and Development, v. 10, n. 4, e2710413724, 2021

(CC BY 4.0) | ISSN 2525-3409 | DOI: http://dx.doi.org/10.33448/rsd-v10i4.13724

Sampaio, J. P. M., Hashimoto, J. M., \& Silva, K. J. D. (2016). Efeitos dos parâmetros de extrusão na solubilidade em água de farinha integral instantânea de feijão-caupi. In: Embrapa Meio- Norte-Artigo em anais de congresso (ALICE). In: Jornada Científica da Embrapa Meio-Norte, 2., 2016, Teresina. Anais da II Jornada Científica da Embrapa Meio-Norte. Teresina: Embrapa Meio-Norte, 2016.

Saubade, F., Hemery, Y. M., Rochette, I., \& Goyot, J-P. (2017). Humbeot. Influence of fermentation and other processing steps on the folate content of a traditional fermented food based on African cereals. Internacional Journal of Food Microbiology, 266, 79-86.

Schiassi, M. C. E. V., De Souza, V. R., lago, A. M. T., Campos, L. G., \& Queiroz, F. (2018). Fruits from the Brazilian Cerrado region: Physico-chemical characterization, bioactive compounds, antioxidant activities, and sensory evaluation. Food chemistry, 245, 305- 311. 10.1016/j.foodchem.2017.10.104

Seixas, F. R. F., Sesquim, E. A. R., Raasch, G. S., \& Cintra, D. E. (2016). Physicochemical characteristics and lipid profile of the bacaba occurring in the western Amazon. Brazilian Journal of Food Research. 7(3), 105-116. 10.3895/rebrapa.v7n3.3806

Selani, M. M., Brazaca, S. G. C., Dias, C. T. S., Ratnayake, W. S., Flores, R. A., \& Bianchini, A. (2014). Characterization and potential application of pineapple pomace in an extruded productfor fibre enhancement. Food Chemistry, 163, 23-30. 10.1016/j.foodchem.2014.04.076

Silveira, M. L. R., Santos, O. S., Penna, N. G., Sautter, C. K., Rosa, C. S., \& Bertagnolli, S. M. M. (2016). Aproveitamento tecnológico das sementes de goiaba (Psidium guajava L.) como farinha na elaboração de biscoitos. Boletim CEPPA, Curitiba-PR, 34(1),1-21.

Sogi, D. S., Siddiq, M., Greiby, I., \& Dolan, K. D. (2013). Fenólicos totais, atividade antioxidante e propriedades funcionais da casca e do caroço de manga 'Tommy Atkins' afetadas por métodos de secagem. Química alimentar, 14(3), 2649-2655.

Tavares, J. A. S., Soares Júnior, M. S., Becker, F. S., \& Costa, E. E. (2012). Mudanças funcionais de farinha de arroz torrada com micro-ondas em função do teor de umidade e do tempo de processamento. Revista Ciência Rural, Santa Maria-RS, 42(6), 1102-1109. 10.1590/S0103-84782012000600025

Wankenne, M. A. (2014). Os tipos e os efeitos da rancidez oxidativa em alimentos. Food Ingredients Brazil, 29, 38-45.

Wycoff, W. Luo, R., Schauss, A. G., Neal-Kababick, J., Sabaa-Srur, A. U., Maia, J. G. S., \& Smith, R. E. (2015). Clerical and nutritional analysis of seeds from purple and white açaí (Euterpe oleracea Mart.). Journal of Food Composition and Analysis, 41, 181-187. 10.1016/j.jfca.2015. 